\title{
Noncommutative BTZ black hole and Fuzzy space
}

\author{
Abderrahman EL Boukili ${ }^{1 *}$, Mourad Nach ${ }^{1}$, Abdellah EL Boukili' ${ }^{2}$ and Moulay Brahim Sedra ${ }^{1}$ \\ ${ }^{I}$ Ibn Tofail University, Faculty of Sciences, Physics Departement, (LHESIR), Kénitra, Morocco. \\ ${ }^{2}$ Mohamed V University, Faculty of Sciences Agdal, (LECA), Rabat, Morocco. \\ *Corresponding author E-mail: aelboukili@gmail.com
}

\begin{abstract}
We study the effects of noncommutative spaces and fuzzy space on the mertic, horizon, entropy and Hawking temperature of a BTZ black hole. The results show deviations from the usual physical properties. The deviations depend on the parameter of noncommutativity.
\end{abstract}

Keywords: BTZ black hole, entropy, fuzzy space, Hawking temperature, horizon.

\section{Introduction}

The BTZ black hole in $(1+1)$ dimensions has been considered before in [1] in the context of models of gravitational theories in $(1+1)$ dimensions. We will follow a less ambitious approach and introduce a model of a BTZ black hole by using the formulation of noncommutative geometry.

In fact, the noncommutative space can be realized by the coordinate operators satisfying [2]

$\left[x_{i}, x_{j}\right]=i \theta_{i j} ; \quad\left[x_{i}, p_{j}\right]=i \delta_{i j} ; \quad\left[p_{i}, p_{j}\right]=0$

where $\theta_{i j}$ is the noncommutativity parameter of dimension (length) 2 . It was noted in [3] that at the replacement

$\boldsymbol{x}_{i}=\hat{\boldsymbol{x}}_{i}+\frac{1}{2} \boldsymbol{\theta}_{i j} \boldsymbol{p}_{j} ; \quad \boldsymbol{p}_{j}=\hat{\boldsymbol{p}}_{j}$

we obtain the standard commutation relations

$$
\left[\hat{\boldsymbol{x}}_{i} ; \hat{\boldsymbol{x}}_{j}\right]=0 ; \quad\left[\hat{\boldsymbol{x}}_{i} ; \hat{\boldsymbol{p}}_{j}\right]=\boldsymbol{i} \boldsymbol{?} \boldsymbol{\delta}_{i j} ; \quad\left[\hat{\boldsymbol{p}}_{i} ; \hat{\boldsymbol{p}}_{j}\right]=0
$$

The usual product of fields should be replaced by the star-product

$$
(f ? g)=\left.\exp \left(\frac{i}{2} \theta_{i j} \frac{\partial}{\partial x^{i}} \frac{\partial}{\partial y^{j}}\right) f(x) g(y)\right|_{x=y}
$$

However, fuzzy space represents another kind of noncommutative geometry, it is known to correspond to the sphere D2-branes in string theory with a linear background B-field [4]. Moreover, in the presence of constant RR field potential, the D0-branes can expand into a noncommutative fuzzy sphere configuration. The quantum field on the fuzzy space has been studied by many authors $[5,6]$. Therefore, it is interesting to see how the fuzzy space will change the black hole property.

The paper is organized as follows. In Sec. 2, we review the main features of BTZ Black hole. In Sec. 3, we study the physical properties of BTZ Black hole in noncommutative space. A brief conclusion is reported in Sec. 4. 


\section{BTZ Black hole}

\section{- The Metric}

The metric of BTZ black hole is given by [7]

$$
d S^{2}=-f(r) d t^{2}+\frac{1}{f(r)} d r^{2}+r^{2} d \phi^{2}
$$

where

$\boldsymbol{f}(\boldsymbol{r})=-\boldsymbol{M}+\frac{\boldsymbol{r}^{2}}{\boldsymbol{l}^{2}}-\frac{\boldsymbol{Q}^{2}}{\boldsymbol{l}^{2}} \ln \left(\frac{\boldsymbol{r}}{\boldsymbol{l}}\right)$

This known as the lapse function and $M$ and $Q$ are the mass and electric charge of the BTZ black hole, respectively.

\section{- Horizon}

For 2 -dimensional BTZ-like solutions, the horizons are located at [8]

$$
\begin{aligned}
& \boldsymbol{r}_{-}=\boldsymbol{l} \exp \left\{-\boldsymbol{L}_{W}\left(\frac{-\boldsymbol{l}^{-1} \exp \left(-\frac{M}{2^{\frac{1}{2}} \boldsymbol{Q}}\right)}{2^{\frac{1}{2}} \boldsymbol{Q}}\right)-\frac{\boldsymbol{M}}{2^{\frac{1}{2}} \boldsymbol{Q}}\right\} \\
& \boldsymbol{r}_{+}=\boldsymbol{l} \exp \left\{-\boldsymbol{L}_{W}\left(-1, \frac{-\boldsymbol{l}^{-1} \exp \left(-\frac{\boldsymbol{M}}{2^{\frac{1}{2}} \boldsymbol{Q}}\right)}{2^{\frac{1}{2}} \boldsymbol{Q}}\right)-\frac{\boldsymbol{M}}{2^{\frac{1}{2}} \boldsymbol{Q}}\right\}
\end{aligned}
$$

where the $L_{W}$ is LambertW function satisfies $L_{W}(x) \exp \left[L_{W}(x)\right]=x$ (for more details, see[9]). The event horizon of presented BTZ-like solutions are located at the $\operatorname{root}(\mathrm{s})$ of $f(r)=0$. Thus, the presented $r \equiv$ is the radius of the event horizon. The BTZ-like solutions may be interpreted as black hole solutions with inner and outer horizons, an extreme black hole or naked singularity.

\section{- Scalar Ricci Curvature}

By definition, the scalar curvature is given by

$$
\begin{aligned}
\boldsymbol{R} & =-\frac{\boldsymbol{d}^{2}}{\boldsymbol{d r}^{2}} \boldsymbol{f}(\boldsymbol{r}) \\
& =\frac{2}{\boldsymbol{l}^{2}}+\frac{\boldsymbol{Q}^{2}}{\boldsymbol{l}^{2}} \frac{1}{\boldsymbol{r}^{2}} \approx \frac{\boldsymbol{Q}^{2}}{\boldsymbol{l}^{2}} \frac{1}{\boldsymbol{r}^{2}}
\end{aligned}
$$

\section{- Electric potential}

The electric potential of this black hole is [10]

$$
\Phi=\frac{\partial \boldsymbol{M}}{\partial \boldsymbol{Q}}=-\boldsymbol{Q} \ln \left(\frac{\boldsymbol{r}}{\boldsymbol{l}}\right)
$$

\section{- Hawking temperature}

while the Hawking temperature is given as[11] 


$$
\begin{aligned}
\boldsymbol{T}_{\boldsymbol{H}} & =\frac{1}{4 \pi}\left|\frac{d \boldsymbol{f}(\boldsymbol{r})}{\boldsymbol{d} \boldsymbol{r}}\right|_{r=r_{+}} \\
& =\frac{\boldsymbol{r}_{+}}{2 \pi \boldsymbol{l}^{2}}-\frac{Q^{2}}{8 \pi} \boldsymbol{r}_{+}
\end{aligned}
$$

\section{- Entropy}

The entropy of BTZ black hole is given by[12]

$$
S_{B T Z}^{B H}=\frac{A}{4}=\frac{\pi}{2} r_{+}
$$

\section{Noncommutative BTZ Black hole}

\section{- Metric}

We have the following metric for BTZ blak hole in non commutative space

$d S^{2}=-f(\tilde{r}) d t^{2}+\frac{1}{f(\tilde{r})} d \tilde{r d} \tilde{r}+\tilde{r} \tilde{r} d \varphi^{2}$

where

$\boldsymbol{f}(\tilde{\boldsymbol{r}})=-\boldsymbol{M}+\frac{\tilde{\boldsymbol{r}} \tilde{\boldsymbol{r}}}{\boldsymbol{l}^{2}}-\frac{\boldsymbol{Q}^{2}}{\boldsymbol{l}^{2}} \ln \left(\frac{\sqrt{\tilde{\boldsymbol{r}} \tilde{\boldsymbol{r}}}}{\boldsymbol{l}}\right)$

where $\tilde{r}$ satisfies following Poisson brackets (3). We note that in a new coordinates systems (2)

one can treat the noncommutative effects as some perturbations of the commutative counter-part, $f(\tilde{r})$ in terms of the noncommutative coordinates $\tilde{x}_{i}$ is as

$f(\tilde{\boldsymbol{r}})=-M+\frac{\tilde{x}_{i} \tilde{x}_{i}}{l^{2}}-\frac{Q^{2}}{l^{2}} \ln \left(\frac{\sqrt{\tilde{x}_{i} \tilde{x}_{i}}}{l}\right)$

Using the new coordinates, we have

$f(\boldsymbol{r})=-M+\frac{\left(x_{i}-\frac{\theta_{i j} p_{j}}{2}\right)\left(x_{i}-\frac{\theta_{k} p_{k}}{2}\right)}{l^{2}}-\frac{Q^{2}}{l^{2}} \ln \left(\frac{\sqrt{\left(x_{i}-\frac{\theta_{i j} p_{j}}{2}\right)\left(x_{i}-\frac{\theta_{k} p_{k}}{2}\right)}}{l}\right)$

where

$\theta_{i j}=\frac{1}{2} \varepsilon_{i j k} \theta_{k}$

therefore

$\boldsymbol{f}(\boldsymbol{r})=-\boldsymbol{M}+\frac{\boldsymbol{r}^{2}}{\boldsymbol{l}^{2}}-\frac{\boldsymbol{Q}^{2}}{\boldsymbol{l}^{2}} \ln \left(\frac{\boldsymbol{r}}{\boldsymbol{l}}\right)-\frac{\overrightarrow{\boldsymbol{L}} \cdot \overrightarrow{\boldsymbol{\theta}}}{4 \boldsymbol{l}^{2}}+\frac{p^{2} \boldsymbol{\theta}^{2}-\overrightarrow{\boldsymbol{p}} \cdot \overrightarrow{\boldsymbol{\theta}}}{16 \boldsymbol{l}^{2}}+\frac{\boldsymbol{Q}^{2}}{16 \boldsymbol{r}^{2}}\left(\overrightarrow{\boldsymbol{L}} \cdot \overrightarrow{\boldsymbol{\theta}}-\frac{p^{2} \boldsymbol{\theta}^{2}-\overrightarrow{\boldsymbol{p}} \cdot \overrightarrow{\boldsymbol{\theta}}}{4}\right)$ 


\section{- Horizon in noncommutative space}

The horizon of the noncommutative metric satisfies the following condition

$$
f(r)=0
$$

therefore the horizons of BTZ black hole in non-commutative space are as following

$$
\begin{aligned}
& \boldsymbol{r}_{-}=l \exp \left\{-\boldsymbol{L}_{W}\left(\frac{-\boldsymbol{l}^{-1} \exp \left(-\frac{M}{2^{\frac{1}{2}}}\right)}{2^{\frac{1}{2}} \boldsymbol{Q}}\right)-\frac{\boldsymbol{M}}{2^{\frac{1}{2}} \boldsymbol{Q}}\right\}+\frac{\overrightarrow{\boldsymbol{L}} \cdot \overrightarrow{\boldsymbol{\theta}}}{8 \boldsymbol{r}_{-}}-\frac{\boldsymbol{p}^{2} \boldsymbol{\theta}^{2}-\vec{p} \cdot \vec{\theta}}{32 \boldsymbol{r}_{-}} \\
& \boldsymbol{r}_{+}=\boldsymbol{l} \exp \left\{-\boldsymbol{L}_{W}\left(-1, \frac{-\boldsymbol{l}^{-1} \exp \left(-\frac{M}{2^{\frac{1}{2}} \boldsymbol{Q}}\right)}{2^{\frac{1}{2}} \boldsymbol{Q}}\right)-\frac{\boldsymbol{M}}{2^{\frac{1}{2}} \boldsymbol{Q}}\right\}+\frac{\overrightarrow{\boldsymbol{L}} \cdot \overrightarrow{\boldsymbol{\theta}}}{8 \boldsymbol{r}_{+}}-\frac{\boldsymbol{p}^{2} \boldsymbol{\theta}^{2}-\overrightarrow{\boldsymbol{p}} \cdot \overrightarrow{\boldsymbol{\theta}}}{32 \boldsymbol{r}_{+}}
\end{aligned}
$$

\section{- Scalar Ricci Curvature in noncommutative space}

The Scalar Ricci Curvature is given by

$$
\begin{aligned}
\boldsymbol{R} & \approx \frac{\boldsymbol{Q}^{2}}{\boldsymbol{l}^{2}} \frac{1}{\tilde{r} \tilde{r}} \approx \frac{\boldsymbol{Q}^{2}}{\boldsymbol{l}^{2}} \frac{1}{\left(\boldsymbol{x}_{\boldsymbol{i}}-\frac{\theta_{i} p_{j}}{2}\right)\left(\boldsymbol{x}_{i}-\frac{\theta_{u} p_{k}}{2}\right)} \\
& \approx \frac{\boldsymbol{Q}^{2}}{\boldsymbol{l}^{2}}\left(\boldsymbol{r}^{2}-\frac{\overrightarrow{\boldsymbol{L}} \cdot \overrightarrow{\boldsymbol{\theta}}}{4}+\frac{\boldsymbol{p}^{2} \boldsymbol{\theta}^{2}-\overrightarrow{\boldsymbol{p}} \cdot \overrightarrow{\boldsymbol{\theta}}}{16}\right)^{-1}
\end{aligned}
$$

\section{- Hawking temperature}

The Hawking temperature $T_{H}$ associated with the outer black hole horizon is

$$
\begin{aligned}
\boldsymbol{T}_{\boldsymbol{H}}= & \frac{\boldsymbol{r}_{+}}{2 \pi \boldsymbol{l}^{2}}-\frac{2 \boldsymbol{Q}^{2}}{\boldsymbol{r}_{+}} \\
& =\frac{1}{2 \pi \boldsymbol{l}} \boldsymbol{\operatorname { e x p }}\left\{-\boldsymbol{L}_{\boldsymbol{W}}\left(-1,-\left(2^{2^{\frac{1}{2}}} \boldsymbol{Q} \boldsymbol{l}\right)^{-1} \exp \left(-\boldsymbol{M}\left(2^{\frac{1}{2}} \boldsymbol{Q}\right)^{-1}\right)\right)-\boldsymbol{M}\left(2^{\frac{1}{2}} \boldsymbol{Q}\right)^{-1}\right\}+\frac{1}{2 \pi \boldsymbol{l}^{2}}\left(\frac{\overrightarrow{\boldsymbol{L}} \cdot \overrightarrow{\boldsymbol{\theta}}}{8 \boldsymbol{r}_{+}}-\frac{\boldsymbol{p}^{2} \boldsymbol{\theta}^{2}-\overrightarrow{\boldsymbol{p}} \cdot \overrightarrow{\boldsymbol{\theta}}}{32 \boldsymbol{r}_{+}}\right) \\
& -2 \boldsymbol{Q}^{2}\left[\boldsymbol{l} \boldsymbol{\operatorname { e x p }}\left\{-\boldsymbol{L}_{\boldsymbol{W}}\left(-1,-\left(2^{\frac{1}{2}} \boldsymbol{Q} \boldsymbol{l}\right)^{-1} \exp \left(-\boldsymbol{M}\left(2^{\frac{1}{2}} \boldsymbol{Q}\right)^{-1}\right)\right)-\boldsymbol{M}\left(2^{\frac{1}{2}} \boldsymbol{Q}\right)^{-1}\right\}+\frac{\overrightarrow{\boldsymbol{L}} \cdot \overrightarrow{\boldsymbol{\theta}}}{8 \boldsymbol{r}_{+}}-\frac{\boldsymbol{p}^{2} \boldsymbol{\theta}^{2}-\overrightarrow{\boldsymbol{p}} \cdot \overrightarrow{\boldsymbol{\theta}}}{32 \boldsymbol{r}_{+}}\right]^{-1}
\end{aligned}
$$

\section{- Entropy}

According to the Bekenstein-Hawking formula, the thermodynamic entropy of a black hole is proportional to the area A of the outer event horizon $\mathrm{S}=\mathrm{A} / 4$. For the charged BTZ black hole we have

$$
\begin{aligned}
\boldsymbol{S} & =\frac{\pi \boldsymbol{r}_{+}}{2} \\
& =\frac{\pi}{2}\left[\boldsymbol{l} \exp \left\{-\boldsymbol{L}_{W}\left(-1,-\left(2^{\frac{1}{2}} \boldsymbol{Q} \boldsymbol{l}\right)^{-1} \exp \left(-\boldsymbol{M}\left(2^{\frac{1}{2}} \boldsymbol{Q}\right)^{-1}\right)\right)-\boldsymbol{M}\left(2^{\frac{1}{2}} \boldsymbol{Q}\right)^{-1}\right\}+\frac{\overrightarrow{\boldsymbol{L}} \cdot \overrightarrow{\boldsymbol{\theta}}}{8 \boldsymbol{r}_{+}}-\frac{\boldsymbol{p}^{2} \boldsymbol{\theta}^{2}-\overrightarrow{\boldsymbol{p}} \cdot \overrightarrow{\boldsymbol{\theta}}}{32 \boldsymbol{r}_{+}}\right]
\end{aligned}
$$

\section{Fuzzy BTZ Black hole}

The static metric ansatz of non-rotating BTZ black hole is described by [13] 
$d s^{2}=-g_{00} d t^{2}+g_{00}^{-1} d r^{2}+r^{2} d \varphi^{2}$

where

$$
g_{00}=\frac{\boldsymbol{r}^{2}}{\ell^{2}}-16 \pi G \int_{0}^{r} d r r \rho_{h}(r)
$$

The matter density is given by the following smeared distribution function [14-16]

$$
\rho_{h}(r)=\frac{M}{2 \pi h^{2}} \exp \left(-\frac{r}{h}\right)
$$

The Hawking temperature $T_{H}$ and the black hole entropy $S$ are calculated by $[17,18]$

$$
\begin{aligned}
T_{H} & \equiv\left(\frac{1}{4 \pi} \frac{d g_{00}}{d r}\right)_{r=r_{H}} \\
S & =\int \frac{d M}{T_{H}}=\int^{r_{H}} \frac{1}{T_{H}} \frac{d M}{d r_{H}} d r_{H}
\end{aligned}
$$

we have to integrate the radius $r_{H}$ from zero, as the minimum radius of fuzzy BTZ black hole is zero.

\section{Conclusion}

In this paper, we have study the effects of noncommutative spaces on the mertic, horizon, entropy and Hawking temperature of a BTZ black hole. Through our paper, the results show deviations from the usual physical properties. The deviations depend on the parameter of noncommutativity and we find the classical results when $\theta$ tends to 0 . However, we have started the study of BTZ black hole in fuzzy space by giving the metric, Hawking temperature and entropy. we will study in detail this part in our next paper.

\section{References}

[1] A. Achucarro and M. Ortiz, Relating black holes in two and three dimensions, Phys. Rev. D, 48 , 3600 (1993) arXiv:hep-th/9304068v1.

[2] S . Doplicher, K. Fred en h agen an d J. E. R ob ert s, C om m un. M ath. Phys. 172, 187 (1995); K . Morit a, Prog. T heor. Phys. 108, 1099 (2003).

[3] S.I. Kruglov, Maxwell's Theory on Non-Commutative Spaces and Quaternions, An-nales Fond.Broglie 27 (2002) 343-358, arXiv:hepth/0110059v2

[4] S. R. Das, S. P. Trivedi, S. Vaidya, Magnetic Moments of Branes and Giant Gravitons, JHEP 0010 (2000) 037

[5] C. Bachas, M. Douglas and C.Schweigert, Brane dynamics in background and noncommutative geometry, JHEP 0005 (2000) 010.

[6] Pei-Ming Ho, Fuzzy Sphere from Matrix Model, JHEP 0012 (2000) 015.

[7] C. Martinez, C. Teitelboim and J. Zanelli. Phys. Rev. D61 (2000) 104013

[8] S. H. Hendi, Charged BTZ-like Black Holes in Higher Dimensions, Eur.Phys.J.C71:1551,2011

[9] R. M. Corless, G. H. Gonnet, D. E. G. Hare, D. J. Jeffrey and D. E. Knuth, Adv. Computational Math. 5. (1996) 329.

[10] Jianyong Shen, Rong-Gen Cai, Bin Wang, Ru-Keng Su, Thermodynamic Geometry and Critical Behavior of Black Holes, Int.J.Mod.Phys.A22:11-27,2007

[11] A. Larrañaga, Entanglement Entropy for the Charged BTZ Black Hole, Bulg. J. Phys. vol.38 no.2 (2011) 123-128.

[12] M. Akbar, H. Quevedo, K. Saifullah, A. Sanchez, S. Taj, Thermodynamic Geometry Of Charged Rotating BTZ Black Holes, Phys.Rev.D83:084031,2011

[13] M. R. Setare, Non-Rotating BTZ Black Hole Area Spectrum from Quasi-normal Modes, Class.Quant.Grav. 21 (2004) 1453-1458

[14] Wung-Hong Huang, : Quantum Field, Thermodynamics and Black Hole on Coherent State Representation of Fuzzy Space, arXiv:1003.1040.

[15] A. EL Boukili, M. Nach, M. B. Sedra, Hawking radiation Schwarzschild black hole in fuzzy space, International Journal of Basic and Applied Sciences, 2 (1) (2013) 81-86

[16] Mourad Nach, Abdellah EL Boukili, Moulay Brahim Sedra and Abderrahman EL Boukili, Anti-de-Sitter Schwarzschild black hole in fuzzy space, International Journal of Basic and Applied Sciences, 2 (1) (2013) 87-90

[17] M. Nach, M. B. Sedra, A. Zemate and A. EL Boukili, Electrically Charged Black Hole Inspired by 2D Fuzzy Sphere, Journal of Modern Physics and Applications, ID599, In press

[18] M. Nach, H. Zaari, M. B. Sedra and A. El Boukili, Charged Rotating Black Hole in 2D Fuzzy Sphere, IJPAP17857, In press 